\title{
PLAN AGREGADO DE PRODUCCIÓN EN BARRACAS MADERERAS. ESTUDIO DE CASO PARA UNA PEQUEÑA INDUSTRIA
}

\section{Aggregated production plan in sawnwood mill. Case of study for small industry}

\author{
Rodrigo del Solar S. ${ }^{1}$, Iván Chacón C. ${ }^{2}$, Mauricio Ponce D. ${ }^{2}$ \\ ${ }^{1}$ Ingeniero Forestal. Bodema Ltda. Talca. Chile \\ ${ }^{2}$ Ingeniero Forestal. Facultad de Ciencias Forestales. Universidad de Talca. Talca. Chile \\ Autor para correspondencia: mponce@utalca.cl \\ Recibido: 24.09. 2007 Aceptado: 07.04. 2008.
}

\section{RESUMEN}

La planificación de la producción en pequeñas empresas es un problema habitual, ya que muchas veces el o los ingenieros no cuentan con el tiempo o las herramientas apropiadas para hacer una buena gestión de la producción. Actualmente, los computadores personales permiten aplicar metodologías de planificación, reduciendo sofisticados problemas en simples estructuras de datos, con apoyo visual de gráficos y tablas, lo que conlleva a un mejor control, tanto de la producción como de los costos. El estudio de caso presentado corresponde al tipo de estas empresas, productora de madera aserrada de pino radiata, en la cual se aplicó una planificación agregada (PA). Se utilizaron datos del último año de producción, y se evaluaron cuatro estrategias productivas relacionadas con la fuerza laboral, el nivel de inventario, de producción y de demanda. Los resultados llevan a concluir que el método propuesto es recomendable para empresas del tamaño estudiado, ya que se puede aplicar fácilmente y permite una adecuada gestión. Con la elección empresarial elegida, fue posible reducir los costos de producción, asegurando políticas laborales y se manejó adecuadamente el nivel de inventario; se redujo en un 1,92\% el costo por pulgada producida, ahorrando la empresa más de 6 millones de pesos anuales.

Palabras Clave: plan agregado, barraca maderera, tasa de producción.

\begin{abstract}
The planning of the production in small-scale industries is a usual problem. Since most of the time, engineers are not provided with the time or the appropriate hardware to do a good management of the production. At the time, personal computers allow to apply methodologies of planning, reducing sophisticated problems in simple structures of data, with visual support of graphs and sheet work, which it bears to a better control, both in the production as it is costs. The study case presented corresponds to the type of these industries, producer of saw wood radiate pine, in which an aggregate production (AP) was applied. There was used information from last year's production, and there were evaluated four productive strategies related to the labor force, the inventory level, production and demand. The results lead to conclude that the proposed method is advisable for companies of the studied size, since it possible to apply easily and allows an appropriate management. With the managerial elected election, it was possible to reduce the costs production, assuring political labor and an appropriately inventory level; the cost diminished in $1.92 \%$ for produced inch, saving the company more than 6 million Chilean pesos annual.
\end{abstract}

Keywords: incorporated plan, small sawnwood mill, production rate. 


\section{INTRODUCCIÓN}

La Planificación Agregada (PA) es la unión entre las decisiones sobre instalaciones y programación, se establecen niveles de producción generales a mediano plazo, políticas de jornadas laborales (contrataciones, despidos, subcontrataciones), nivel de inventarios, considerando la demanda, los flujos de insumos, precios y costos, como las variables más importantes para su aplicación. Buxey (2005) señala que en Estados Unidos no hay evidencia que la industria la haya adoptado ampliamente después de más de 50 años de desarrollo y, si se aplica, se hace con modificaciones. Gunasekaran y Marri (2004) estudiaron la PA en países en desarrollo como India, Malasia, México y Brasil, encontrando que no ha tenido la atención que se esperaba, considerando el desarrollo de las tecnologías de información.

La PA se ha aplicado a una amplia variedad de industrias. En el ámbito forestal se utiliza para la planificación de actividades de mediano plazo en la industria de la celulosa y papel (Martel et al. 2004). En aserraderos, Pradenas et al. (2004) propuso una serie de soluciones factibles como modelo heurístico, aún cuando no hay evidencia de su aplicación práctica. Por ello, sigue siendo un desafío en los sistemas de planificación y control de la producción (Mula et al. 2006).

Según Adam y Ebert (1991) el diseño de la PA requiere identificar una medida significativa de producción de las líneas de productos, que compartan procesos de producción comunes o consuman similares recursos. Chase et al. (2005) señalan que al menos es necesario identificar tres estrategias para la elaboración: a) el tamaño de la fuerza laboral, b) el inventario y c) el volumen de trabajo atrasado.

Welsch (2005) indica que un plan de producción no tiene por objetivo determinar las cantidades precisas y los tiempos de producción reales en el período presupuestado, sino que busca representar las derivaciones del volumen planificado de las ventas para el volumen proyectado de producción, como base de las necesidades de capacidad de la planta, de materiales y de componentes directos, como compras, mano de obra y gastos indirectos de fabricación. En tal sentido Monk (1992) indica que los pronósticos son estimaciones de ocurrencia. Su objetivo es usar de mejor forma la información disponible para guiar actividades tendientes al cumplimiento de las metas de la organización, como por ejemplo la demanda; aunque también son importantes el precio de la materia prima, los costos de mano de obra, las tasas de interés y los ingresos.

Schroeder (1992) plantea que se pueden utilizar dos estrategias para satisfacer las fluctuaciones de la demanda en el tiempo. Una es nivelar la fuerza de trabajo, y la otra relacionar la demanda con la fuerza de trabajo. Con ello habrá constancia en la producción en tiempo normal y cualquier variación en la demanda podrá paliarse mediante el uso de inventarios o de otras variables que influyen en la demanda.

En otro aspecto, Heizer y Render (2001) indican que una razonable calidad de vida en el trabajo no sólo es adecuadamente segura y se paga un salario justo, sino que alcanza un nivel apropiado de requerimientos físicos y psicológicos. Un compromiso y confianza mutuos significa que dirección y empleados luchan por alcanzar objetivos comunes, con políticas de empleo razonables y documentadas, implementadas honesta y equitativamente para todos.

Los inventarios son considerados recursos ociosos que poseen un valor económico. Las empresas generalmente los clasifican como: 1) materias primas, 2) productos en proceso, los que en ocasiones se acumulan en varios puntos del proceso (Noori y Radford, 1997) y 3) productos terminados. Mantenerlos consume capital, el cual puede no estar proporcionando dividendos a la inversión, por consiguiente el desafío de su administración es mantenerlos a niveles adecuados, pero no excesivos (Monks, 1992).

La mayor influencia sobre los costos de los productos y servicios está dada por la selección que hace el administrador en cuanto a la mezcla de productos, diseño, desempeño, calidad, características especiales, distribución, entre otros. Cada una de estas decisiones contribuye al desempeño de la organización en un marco de evaluación de costo y beneficio (Horngren y Sundem, 1994). Asimismo, la definición del precio de venta debe conciliar diversas variables que influyen sobre el comportamiento del mercado. En primer lugar, está la demanda asociada a distintos niveles de precio, luego los de la competencia para productos iguales y sustitutos y, por último, los costos (Sapag y Sapag, 1996).

No hay publicaciones de experiencias concretas de aplicación de PA en aserraderos. Pradenas et al. (2004) evaluó un modelo de programación heurística con datos de un aserradero mediano utilizando lenguaje $\mathrm{C}++$; con los siguientes elementos de análisis: 9 tipos de productos, 10 formas de ahusamiento de los 
troncos y 10 esquemas de corte. Se han aplicado en otros ámbitos como la construcción (Jingsheng, 1999) en la industria de la pulpa y el papel (Bredstrom et al. 2004; Martel et al. 2004) y en cambios de demanda estacional de la industria (Buxley, 2003).

El objetivo del estudio fue aplicar PA que satisfaga la demanda esperada, con los costos operativos mínimos a lo largo de un horizonte de doce meses, manteniendo un nivel laboral permanente mínimo. Considerando que la PA se plantea como un ejercicio de optimización, en la mayoría de los casos con niveles de dificultad altos, los ingenieros de estas industrias deben realizar diversas tareas en todo el proceso productivo y tienen escaso tiempo para la planificación. En tal caso, el estudio se presenta como una herramienta efectiva y viable para dar soporte a la planificación de la producción de manera simple y confiable, permitiendo reducir costos y aplicar diversas políticas empresariales.

El artículo se estructura con la identificación y los antecedentes aportados por la empresa para la PA, como nivel de producción, costos de producción, inventarios y fuerza laboral. Se evalúan 4 propuestas de trabajo relacionadas con las variables antes mencionadas, y se determina cuál es la mejor opción de acuerdo con el compromiso de la empresa en términos laborales.

\section{MATERIAL Y MÉTODOS}

La unidad de estudio es una empresa maderera ubicada en la ciudad de Talca, Región del Maule, Chile. Su producción alcanza las 188 mil pulgadas anuales de pino radiata, destinadas a productos elaborados y dimensionados secos para el mercado de la construcción. La materia prima corresponde a basas, las cuales son almacenadas y enviadas luego al despuntador que las dimensiona a largo estándar de 3,2 metros. Luego, son dimensionadas a diferentes escuadrías, dirigidas al patio y ubicadas en castillos para su secado durante un período variable, dependiendo de la estación y las condiciones atmosféricas, proceso que puede durar de 10 a 90 días. A continuación moldurado y cepillado, para finalmente, almacenar el producto en bodegas de la empresa. La comercialización se realiza de acuerdo a la calidad, destinándose la venta de productos calificados como extra a distribuidores mayoristas, el saldo correspondiente a las calidades primera, segunda y tercera que son vendidas a minoristas, constructoras, industriales y venta al detalle en la ciudad. En la figura 1 se puede observar el "layout" de la maquinaria en la empresa.

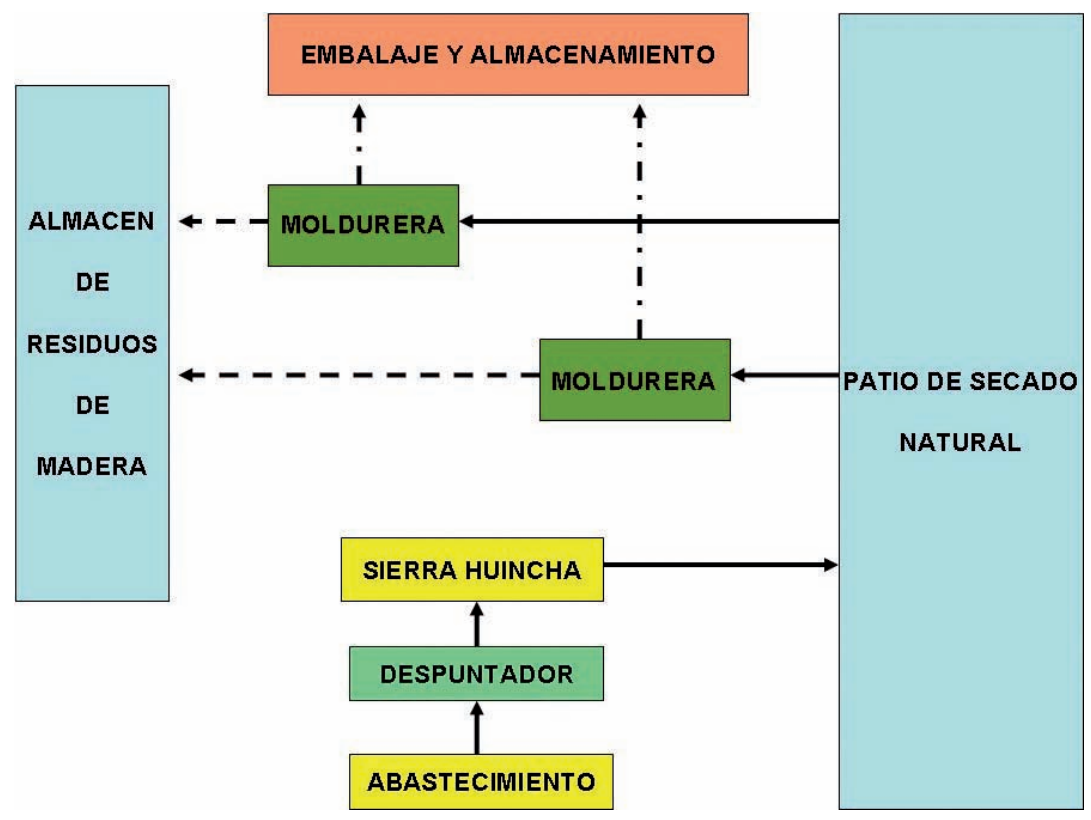

Figura 1. Distribución de máquinas en la empresa. 
Para diseñar un plan agregado fue necesario identificar una medida significativa de producción que tuviera sentido en el contexto, para ello se usó la "pulgada pinera". Asimismo, se empleó un método gráfico para ayudar a encontrar un plan que satisfaga la demanda esperada, con los costos operativos mínimos a lo largo de un horizonte de doce meses.

El modelo matemático de optimización implícito que se desarrolla es:

$$
\operatorname{Max} \Re=\sum_{i=1}^{t}\left(\sum_{k=1}^{k} I_{t k}-\sum_{m=1}^{m} C_{m t}-\sum_{k=1}^{k} L_{k t}-\sum_{k=1}^{k} M_{k t}\right)
$$

Donde:

$\mathrm{I}_{\mathrm{kt}}=$ Ingreso por producto $\mathrm{k}$ en el tiempo $t$

$\mathrm{C}_{\mathrm{kt}}=$ Costo inmóvil del producto $\mathrm{k}$ en el tiempo $t^{2}$

$\mathrm{L}_{\mathrm{kt}}=$ Costo de la mano de obra por producto $k$ en el tiempo $t$

$\mathrm{M}_{\mathrm{kt}}=$ Costo del inventario por producto $k$ en el tiempo $t$

Las restricciones asociadas al modelo son:

a) La demanda debe ser cubierta todo el periodo de planificación

b) Mantener un inventario del 10\% sobre la demanda

c) Una fuerza laboral de contrato permanentemente máxima, al menor costo.

d) Producción constante

e) Límite de la producción acorde con la capacidad instalada

En la PA se consideraron los siguientes antecedentes: a) recopilación y tabulación de datos obtenidos en el período referencial, que va de octubre de 2004 a septiembre de 2005, el que incluye la capacidad de las instalaciones, demanda, procesos productivos, fuerza laboral, productividad, inventarios y costos; b) análisis de las tasas proyectadas de demanda, producción, productividad, fuerza laboral e inventarios para el período planificado; c) propuesta de cuatro planes alternativos al período referencial, basado en una combinación de las variables fuerza laboral e inventario, ambas necesarias para satisfacer la demanda pronosticada, con una tasa de producción constante; d) cálculo, tabulación y gráfico de las variables de demanda, procesos productivos, inventario y fuerza laboral para cada plan propuesto; e) evaluación para cada uno de los planes propuestos de los costos operativos de cada plan; y f) comparación de los planes y elección del plan agregado de acuerdo a los criterios iniciales.

Las estrategias basadas en combinaciones de las variables fuerza laboral e inventarios (se consideró por política de la empresa, que la fuerza laboral es una variable significativa para la planificación, manteniendo un número máximo de obreros permanentes con independencia del nivel de demanda y producción, pero al menor costo), son:

a) Plan 1. Fuerza laboral e inventarios constante, cuando la producción mensual disminuye se debe mantener los niveles de empleo constantes. Esto supone tener una fuerza de trabajo formada y mantener los costos de contratación y despidos al mínimo.

b) Plan 2. Fuerza laboral ajustada a la producción e inventario constante, cuando la producción disminuye se pueden despedir empleados y cuando crece se puede aumentar la fuerza laboral de acuerdo con ese aumento.

c) Plan 3. Fuerza laboral constante e inventario ajustado a la producción, cuando es posible acumular el inventario para su uso posterior durante períodos de menor demanda. En consecuencia el inventario desacopla la oferta de la demanda en las operaciones de manufactura, permitiendo una operación más uniforme.

d) Plan 4. Fuerza laboral e inventarios ajustados a la producción cuando, el manejo del inventario ajustado a la producción representa una complicación adicional en lo que a logística se refiere. Un problema de

Adaptado de Pradenas et al. 2004

Costo inmóvil corresponde a los costos de administración, materias primas, electricidad, grúas, insumos, mantenimiento y herramientas. 
la administración es el mantenimiento de inventarios adecuados, es decir, sin exceso y sin escasez.

En la tabla 1, se muestra la capacidad de producción de acuerdo con los distintos productos a procesar, considerando la dotación trabajadores y máquinas disponibles.

Tabla 1. Capacidad instalada mensual

\begin{tabular}{|c|c|c|c|c|}
\hline \multirow{2}{*}{ Proceso } & \multicolumn{4}{|c|}{ Volumen (pulg/mes) } \\
\cline { 2 - 5 } & Mínimo & Intermedio & Máximo & Promedio \\
\hline Dimensionado & 16.595 & 21.336 & 28.907 & 22.279 \\
\hline Elaborado & 14.520 & 18.669 & 25.293 & 19.494 \\
\hline
\end{tabular}

A fin de utilizar el total de la capacidad instalada de la empresa, se requiere una fuerza laboral de 9 personas en producción directa. Ésta se distribuye entre el dimensionado con 5 personas y el elaborado con 4. La capacidad de producción del secado depende del número de empleados destinados a esa labor.

Para construir un pronóstico de venta que permita proyectar los niveles de producción y variables asociadas, como nivel de fuerza laboral e inventarios, se cuantificó la demanda durante el período referencial. Asimismo, se estableció la tasa de producción por grupo de productos en el periodo referencia, que se observan en la tabla 2 .

Tabla 2. Demanda y producción del periodo referencial (pulgadas)

\begin{tabular}{|l|c|c|r|c|c|r|}
\hline \multirow{2}{*}{ Mes } & \multicolumn{3}{|c|}{ Demanda } & \multicolumn{3}{c|}{ Proceso productivo } \\
\cline { 2 - 7 } & Dimensionado & Elaborado & \multicolumn{1}{c|}{ Total } & Dimensionado & Elaborado & Secado \\
\hline Octubre & 1.772 & 8.463 & 10.235 & 24.227 & 14.800 & 11.158 \\
\hline Noviembre & 1.360 & 13.907 & 15.267 & 20.149 & 18.150 & 17.125 \\
\hline Diciembre & 3.803 & 16.807 & 20.610 & 21.858 & 22.800 & 21.086 \\
\hline Enero & 4.675 & 17.084 & 21.759 & 21.601 & 21.828 & 18.380 \\
\hline Febrero & 1.209 & 12.793 & 14.002 & 22.904 & 18.095 & 15.021 \\
\hline Marzo & 3.195 & 15.959 & 19.154 & 21.932 & 17.300 & 20.925 \\
\hline Abril & 3.302 & 15.230 & 18.532 & 25.720 & 16.950 & 14.373 \\
\hline Mayo & 3.278 & 8.080 & 11.358 & 9.706 & 6.250 & 7.656 \\
\hline Junio & 1.528 & 12.714 & 14.242 & 10.603 & 4.900 & 7.127 \\
\hline Julio & 725 & 7.749 & 8.474 & 5.449 & 5.250 & 10.076 \\
\hline Agosto & 1.539 & 6.143 & 7.682 & 1.685 & 2.150 & 7.452 \\
\hline Septiembre & 2.002 & 8.620 & 10.622 & 2.161 & 7.600 & 9.150 \\
\hline Total & 28.388 & 143.549 & 171.937 & 187.995 & 156.073 & 159.529 \\
\hline
\end{tabular}

En la tabla 3 se presentan la fuerza laboral y la productividad durante el período; la mano de obra es estable para el dimensionado y elaborado, pero para el secado es subcontratada. El promedio de la productividad se obtuvo ponderando respecto del volumen. No existió una estrategia de recursos humanos, actuándose en forma intuitiva respecto al número de empleados, tipos de contrato y horas extras. La fuerza laboral alcanzó una productividad de 20,9 pulg/hh en el dimensionado y 17,4 pulg/hh en cepillado. En consideración a lo anterior, se estableció que la fuerza laboral estable será la que produzca en promedio $21,5 \mathrm{pulg} / \mathrm{hh}$ para madera dimensionada y 19,5 pulg/hh para elaboradas, mientras que para el secado se estimó en $30 \mathrm{pulg} / \mathrm{hh}$ bajo la modalidad de subcontrato. 
Tabla 3. Fuerza laboral y productividad del periodo referencial

\begin{tabular}{|l|c|c|r|r|r|r|}
\hline \multirow{2}{*}{ Mes } & \multicolumn{3}{|c|}{ Fuerza laboral (hh) } & \multicolumn{3}{c|}{ Productividad (pulg/hh) } \\
\cline { 2 - 7 } & Dimensionado & Elaborado & Secado & Dimensionado & Elaborado & Secado \\
\hline Octubre & 1.207 & 792 & 493 & 23,6 & 14,1 & 30,0 \\
\hline Noviembre & 1.023 & 840 & 605 & 19,7 & 20,4 & 30,0 \\
\hline Diciembre & 946 & 871 & 760 & 23,1 & 24,2 & 30,0 \\
\hline Enero & 1.020 & 796 & 727 & 21,2 & 23,1 & 30,0 \\
\hline Febrero & 881 & 736 & 603 & 26,0 & 20,4 & 30,0 \\
\hline Marzo & 850 & 764 & 577 & 25,8 & 27,4 & 30,0 \\
\hline Abril & 874 & 692 & 565 & 29,4 & 20,8 & 30,0 \\
\hline Mayo & 489 & 740 & 208 & 19,8 & 10,3 & 30,0 \\
\hline Junio & 451 & 688 & 163 & 23,5 & 10,4 & 30,0 \\
\hline Julio & 496 & 772 & 174 & 11,0 & 13,1 & 30,0 \\
\hline Agosto & 484 & 760 & 72 & 3,5 & 9,8 & 30,0 \\
\hline Septiembre & 452 & 736 & 254 & 4,8 & 12,4 & 30,0 \\
\hline Total & 8.993 & 9.187 & 5.200 & 20,9 & 17,4 & 30,0 \\
\hline
\end{tabular}

Nota: El total para productividad corresponde al promedio ponderado.

Las maderas en sus distintas formas o grados de elaboración que mantuvo la empresa para satisfacer la demanda del mercado durante el período, se presenta en la tabla 4.

Tabla 4. Inventarios de madera en período referencial (pulgadas)

\begin{tabular}{|c|c|c|c|c|c|c|c|c|c|c|c|c|c|}
\hline \multirow{2}{*}{ Forma } & \multicolumn{13}{|c|}{ MES año $2004-2005$} \\
\hline & Oct. & Nov. & Dic. & Ene. & Feb. & Mar. & Abr. & May. & Jun. & Jul. & Ago. & Sep. & Promedio \\
\hline Mat. Prima & 3.893 & 4.070 & 0 & 1.383 & 1.267 & 2.698 & 174 & 0 & 2.778 & 2.059 & 0 & 0 & $1.526,8$ \\
\hline En proceso & 13.830 & 25.127 & 26.971 & 23.780 & 22.306 & 28.980 & 26.792 & 36.337 & 33.609 & 35.557 & 30.205 & 22.899 & $27.199,4$ \\
\hline Producto & 4.548 & 5.557 & 6.411 & 9.461 & 13.135 & 8.678 & 12.629 & 12629 & 12302 & 6.109 & 8.105 & 12.184 & $9.299,2$ \\
\hline
\end{tabular}

En la tabla 5 se muestra el costo durante el período referencial.

Tabla 5. Costos del período referencial (miles \$)

\begin{tabular}{|c|c|c|c|c|c|c|c|c|c|c|c|c|c|}
\hline \multirow{2}{*}{ Ítems } & \multicolumn{13}{|c|}{ MES año $2004-2005$} \\
\hline & Oct. & Nov. & Dic. & Ene. & Feb. & Mar. & Abr. & May. & Jun. & Jul. & Ago. & Sep. & Total \\
\hline Administración & 2.052 & 1.784 & 1.855 & 1.748 & 1.831 & 1.854 & 2.235 & 2.180 & 1.922 & 1.807 & 1.856 & 1.786 & 22.911 \\
\hline Mat. Prima & 30.284 & 25.186 & 27.323 & 27.001 & 28.630 & 27.415 & 32.150 & 12.133 & 13.254 & 6.811 & 2.106 & 2.701 & 234.994 \\
\hline E. Eléctrica & 755 & 934 & 993 & 953 & 845 & 990 & 909 & 540 & 549 & 472 & 429 & 540 & 8.906 \\
\hline Grúas & 660 & 625 & 664 & 602 & 631 & 598 & 610 & 591 & 527 & 633 & 533 & 569 & 7.268 \\
\hline Insumo: & 210 & 158 & 168 & 188 & 123 & 128 & 238 & 66 & 74 & 61 & 36 & 12 & 1.462 \\
\hline Mant & 390 & 339 & 565 & 453 & 586 & 235 & 255 & 414 & 276 & 513 & 273 & 237 & 4.536 \\
\hline Herram & 438 & 42 & 0 & 0 & 0 & 0 & 448 & 0 & 0 & 65 & 0 & 102 & 1.095 \\
\hline & 267 & 329 & 341 & .238 & 189 & 255 & 215 & 931 & 883 & 961 & 52 & 923 & 13.485 \\
\hline 2. Event & 222 & 146 & 71 & 152 & 140 & 85 & 85 & 89 & $\begin{array}{r}63 \\
62\end{array}$ & $\begin{array}{r}301 \\
91\end{array}$ & 81 & 63 & 1.287 \\
\hline 3. Horas extr: & 30 & 108 & 143 & 176 & 20 & 0 & 0 & 0 & 0 & 0 & 0 & 0 & 477 \\
\hline 4. Bonos & 136 & 157 & 188 & 191 & 300 & 241 & 129 & 161 & 245 & 308 & 318 & 329 & 2.704 \\
\hline 5. Subcontratos & 620 & 761 & 956 & 915 & 759 & 726 & 711 & 262 & 205 & 219 & 91 & 320 & 6.543 \\
\hline 6. Contratos & 0 & 0 & 0 & 0 & 0 & 0 & 0 & 0 & 0 & 150 & 0 & 0 & 150 \\
\hline 7. Despidos & 0 & 0 & 0 & 0 & 0 & 300 & 0 & 600 & 0 & 0 & 300 & 0 & 1.200 \\
\hline Inventarios & 350 & 568 & 575 & 540 & 730 & 705 & 35 & 442 & 597 & 820 & 617 & 743 & 6.723 \\
\hline
\end{tabular}


Para la elaboración de los datos de la tabla 5 se tuvieron las siguientes consideraciones: a) infraestructura y capital propios; b) precios actualizados al 30 de Septiembre de 2005; c) costo de la mano de obra calculado multiplicando las horas por el valor según modalidad de contrato, de acuerdo a: estable de 830 $\$ / \mathrm{h}$ promedio (no considera hora extra); eventual de $830 \$ / \mathrm{h}$ del personal no estable o subcontratado; hora extra $1.245 \$ / \mathrm{h}$, trabajadas por personal estable; bonos, correspondiente al pago adicional por resultado pactado según labor; subcontrato por $1.258 \$ / \mathrm{h}$, pagada a un subcontratista para producir las unidades, que pueden ser mayores, iguales o menores al costo de la producción de las unidades internas, que corresponde, en este caso, a las horas trabajadas en el proceso de secado; costo de contratación $\$ 150.000$, que incluye los costos de reclutamiento, selección y capacitación para cubrir una vacante totalmente capacitada y productiva; costo de despido de $\$ 300.000$, que incluye las prestaciones del personal, prima de antigüedad y otros costos relacionados con el despido; d) el costo de inventario se calculó multiplicando el diferencial entre el inventario del periodo y el inventario ajustado a la demanda del mismo, por el valor asignado según se encuentra el inventario. Para el cálculo de los costos de inventario, los valores considerados son: i) excedente, se consideraron los costos de mantenimiento como un $2 \%$ del volumen inmovilizado; estos costos se relacionan con mantener productos, e incluyen el costo de capital, el costo variable de almacenamiento, el costo de obsolescencia y de deterioro; ii) escasez, se castigó el incumplimiento de algún pedido con un $4 \%$ del volumen, costo difícil de estimar, pero puede relacionarse con la pérdida de prestigio y la pérdida de venta futura.

Los costos promedios considerados según su grado de elaboración fueron: a) materia prima $\$ 1.250$, en proceso $\$ 1.450$ y producto terminado $\$ 1.650$. Finalmente, el costo unitario promedio del periodo fue de $1.669 \$$ pulg.

En la tabla 6 se muestra un resumen con los datos de los volúmenes obtenidos durante el período referencial, los que se utilizaron para la confección del gráfico, según da cuenta la figura 2.

Tabla 6. Datos período referencial

\begin{tabular}{|c|c|c|c|c|c|c|c|c|c|c|c|c|c|}
\hline \multirow{2}{*}{ İtem } & \multicolumn{9}{|c|}{ MES año 2004-2005 } \\
\cline { 2 - 13 }$y$ & Oct. & Nov. & Dic. & Ene. & Feb. & Mar. & Abr. & May. & Jun. & Jul. & Ago. & Sep. & Media \\
\hline Días productivos & 24 & 24 & 26 & 24 & 23 & 24 & 24 & 24 & 24 & 24 & 25 & 24 & 24 \\
\hline Demanda (pulg.) & 10.235 & 15.267 & 20.610 & 21.759 & 14.002 & 19.154 & 18.532 & 11.358 & 14.242 & 8.474 & 7.682 & 10.622 & 14.328 \\
\hline Producción (pulg.) & 24.227 & 20.149 & 21.858 & 21.601 & 22.904 & 21.932 & 25.720 & 9.706 & 10.603 & 5.449 & 1.685 & 2.161 & 15.666 \\
\hline Inventario (pulg.) & 22.271 & 34.754 & 33.382 & 34.624 & 36.708 & 40.356 & 39.595 & 48.808 & 48.689 & 43.725 & 38.310 & 35.083 & 38.025 \\
\hline Fuerza laboral (hr/h) & 21.464 & 21.381 & 19.771 & 21.318 & 18.413 & 17.765 & 18.267 & 10.220 & 9.426 & 10.366 & 10.116 & 9.447 & 15.663 \\
\hline
\end{tabular}




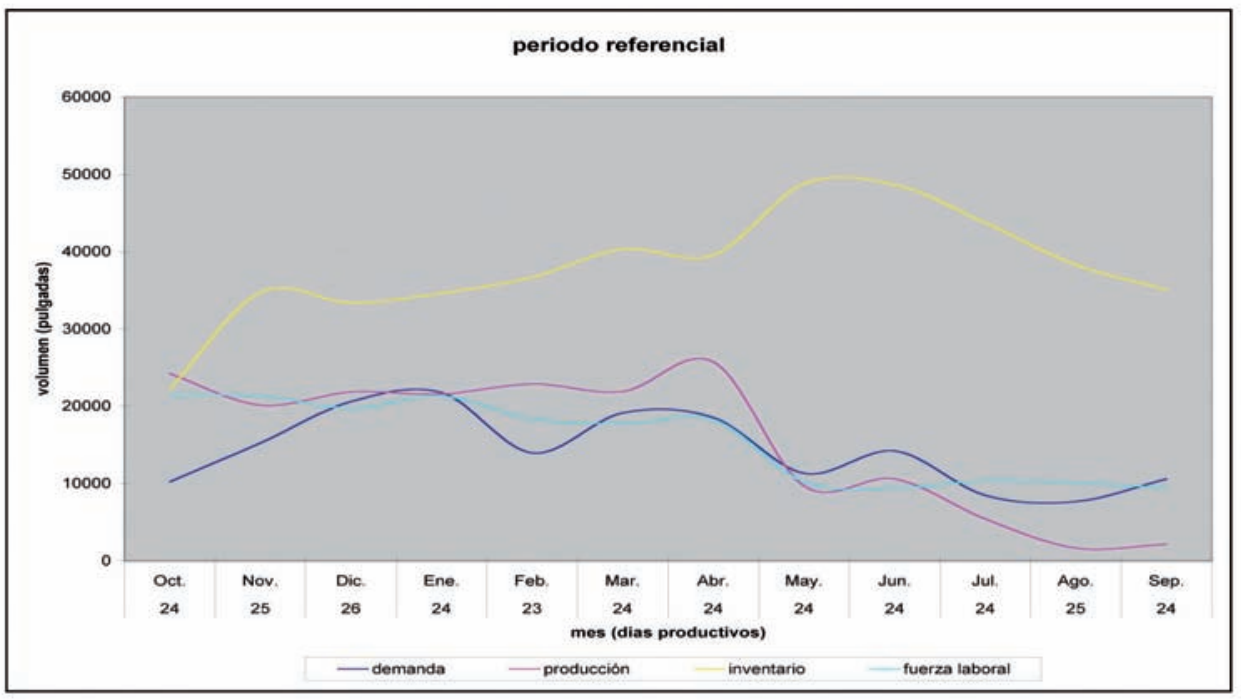

Figura 2. Gráfico de volúmenes en periodo referencial (pulgadas)

La tasa de producción mensual para el período a planificar se proyectó asumiendo una demanda similar a la del período referencial, más un $10 \%$ de producción mensual como inventario de seguridad. Solo fueron considerados los productos que requieren dimensionado y elaborado.

Como soporte computacional para cálculos y gráfica, se utilizó Excel para Windows.

\section{RESULTADOS}

La tabla 7 muestra los resultados de los volúmenes proyectados para el período.

Tabla 7. Tasa de demanda y producción proyectadas (pulgadas)

\begin{tabular}{|l|c|c|c|c|c|r|}
\hline \multirow{2}{*}{ Mes } & \multicolumn{3}{|c|}{ Demanda } & \multicolumn{3}{c|}{ Producción } \\
\cline { 2 - 7 } & Dimensionado & Elaborado & \multicolumn{1}{c|}{ Total } & Dimensionado & Secado & Elaborado \\
\hline Octubre & 1.772 & 8.463 & 10.235 & 11.259 & 11.259 & 9.309 \\
\hline Noviembre & 1.360 & 13.907 & 15.267 & 16.794 & 16.794 & 15.298 \\
\hline Diciembre & 3.803 & 16.807 & 20.610 & 22.671 & 22.671 & 18.488 \\
\hline Enero & 4.675 & 17.084 & 21.759 & 23.935 & 23.935 & 18.792 \\
\hline Febrero & 1.209 & 12.793 & 14.002 & 15.402 & 15.402 & 14.072 \\
\hline Marzo & 3.195 & 15.959 & 19.154 & 21.069 & 21.069 & 17.555 \\
\hline Abril & 3.302 & 15.230 & 18.532 & 20.385 & 20.385 & 16.753 \\
\hline Mayo & 3.278 & 8.080 & 11.358 & 12.494 & 12.494 & 8.888 \\
\hline Junio & 1.528 & 12.714 & 14.242 & 15.666 & 15.666 & 13.985 \\
\hline Julio & 725 & 7.749 & 8.474 & 9.321 & 9.321 & 8.524 \\
\hline Agosto & 1.539 & 6.143 & 7.682 & 8.450 & 8.450 & 6.757 \\
\hline Septiembre & 2.002 & 8.620 & 10.622 & 11.684 & 11.684 & 9.482 \\
\hline Total & 28.388 & 143.549 & 171.937 & 189.131 & 189.131 & 157.904 \\
\hline
\end{tabular}


La estimación de la productividad promedio se presenta en la tabla 8. Se determinó en función de las necesidades que se relacionan con tiempo extra, contrataciones, despidos y subcontrataciones, para proponer el nivel de la fuerza laboral necesario para cumplir con los niveles de producción propuestos. Por otro lado, los niveles de inventarios propuestos en el plan agregado se obtuvieron de la información referida a los volúmenes mensuales de demanda proyectada más un 10\% de seguridad, que se muestran en la tabla 9. Para proyectar un inventario que cumpla con los requerimientos de demanda del producto, es necesario conocer los tiempos de secado de la madera según la estacionalidad y programar los volúmenes a secar en función de esos tiempos.

Tabla 8. Productividad y fuerza laboral proyectada.

\begin{tabular}{|c|c|c|c|c|c|c|}
\hline \multirow{3}{*}{ Mes } & \multicolumn{3}{|c|}{ Productividad (pulg/hh) } & \multicolumn{3}{|c|}{ Fuerza laboral (hh) } \\
\hline & \multicolumn{2}{|c|}{ Estable } & \multirow{2}{*}{$\begin{array}{c}\text { Subcontrato } \\
\text { Secado }\end{array}$} & \multicolumn{2}{|c|}{ Estable } & \multirow{2}{*}{$\begin{array}{c}\text { Subcontrato } \\
\text { Secado }\end{array}$} \\
\hline & Dimensionado & Elaborado & & Dimensionado & Elaborado & \\
\hline Octubre & 23,6 & 14,1 & 30,0 & 523 & 477 & 375 \\
\hline Noviembre & 19,7 & 20,4 & 30,0 & 780 & 784 & 560 \\
\hline Diciembre & 23,1 & 24,2 & 30,0 & 1.054 & 948 & 756 \\
\hline Enero & 21,2 & 23,1 & 30,0 & 1.112 & 963 & 798 \\
\hline Febrero & 26,0 & 20,4 & 30,0 & 716 & 721 & 513 \\
\hline Marzo & 25,7 & 27,4 & 30,0 & 979 & 900 & 702 \\
\hline Abril & & 20,8 & 30,0 & 947 & 859 & 679 \\
\hline Mayo & 19,8 & & 30,0 & 581 & 456 & 416 \\
\hline Junio & 23,5 & & 30,0 & 728 & 717 & 522 \\
\hline Julio & 11,0 & 13,1 & 30,0 & 433 & 437 & 311 \\
\hline Agosto & & & 30,0 & 393 & 346 & 282 \\
\hline Septiembre & & & 30,0 & 543 & 486 & 389 \\
\hline Promedio & 21,5 & 19,5 & 30,0 & 732 & 675 & 525 \\
\hline
\end{tabular}

Tabla 9. Inventario proyectado (pulgadas)

\begin{tabular}{|c|c|c|c|c|c|c|c|c|c|c|c|c|c|}
\hline \multirow{2}{*}{$\begin{array}{c}\text { ETAPA } \\
\text { Materia prima }\end{array}$} & \multicolumn{10}{|c|}{ MES } \\
\cline { 2 - 16 } & Oct. & Nov. & Dic. & Ene. & Feb. & Mar. & Abr. & May. & Jun. & Jul. & Ago. & Sep. & Promedio \\
\hline En proceso & 1.235 & 15.267 & 14.052 & 16.578 & 12.602 & 16.542 & 38.453 & 34.074 & 28.652 & 16.156 & 17.292 & 10.622 & 19.210 \\
\hline Producto & 0 & 0 & 0 & 0 & 0 & 0 & 0 & 0 & 0 & 0 & 0 & 0 & 0 \\
\hline seguridad 10\% & 1.024 & 1.527 & 1.405 & 1.658 & 1.260 & 1.654 & 3.845 & 3.407 & 2.865 & 1.616 & 1.729 & 1.062 & 1.921 \\
\hline Total & 11.259 & 16.794 & 15.457 & 18.236 & 13.862 & 18.196 & 42.298 & 37.481 & 31.517 & 17.772 & 19.021 & 11.684 & 21.131 \\
\hline
\end{tabular}

En los siguientes gráficos se muestran los resultados de los cuatro planes propuestos. 


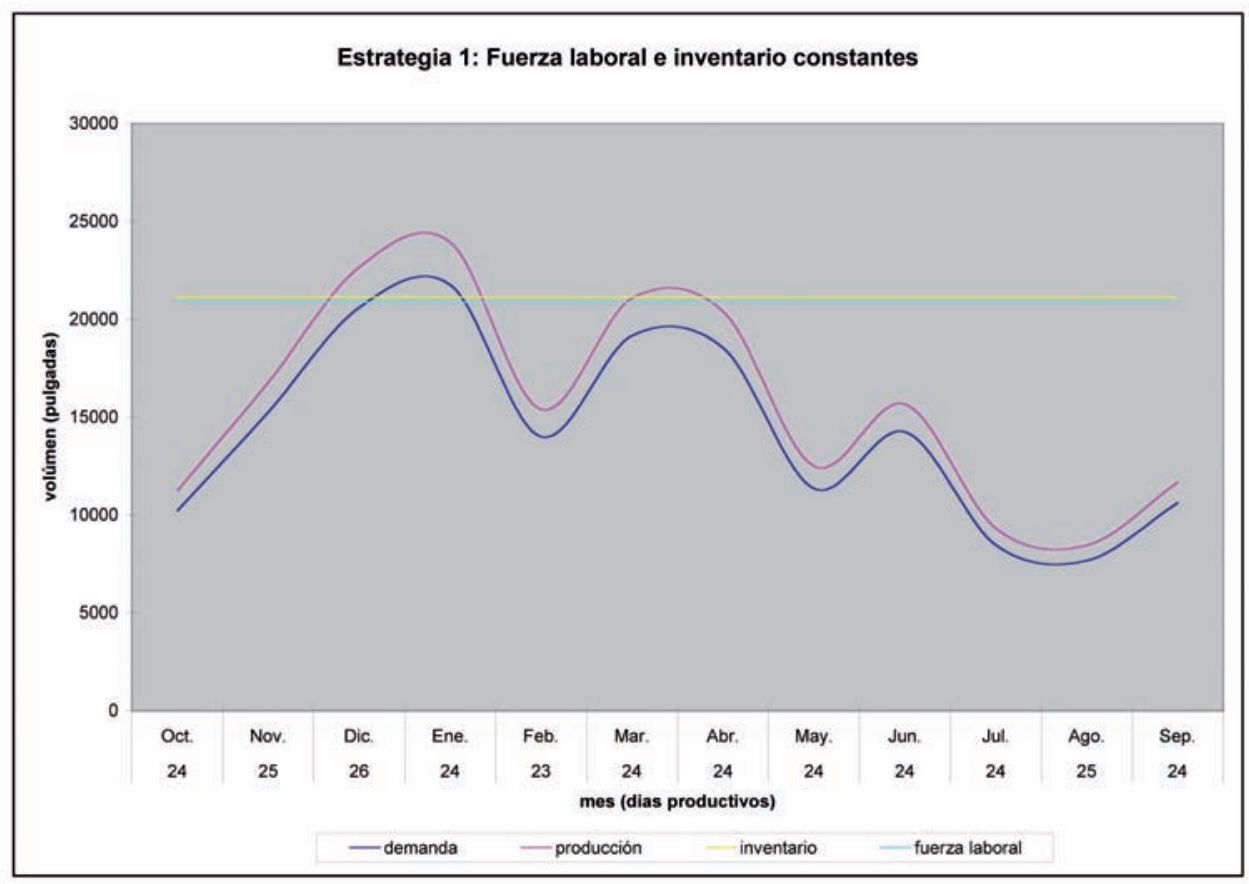

Figura 3. Gráfico Plan 1.

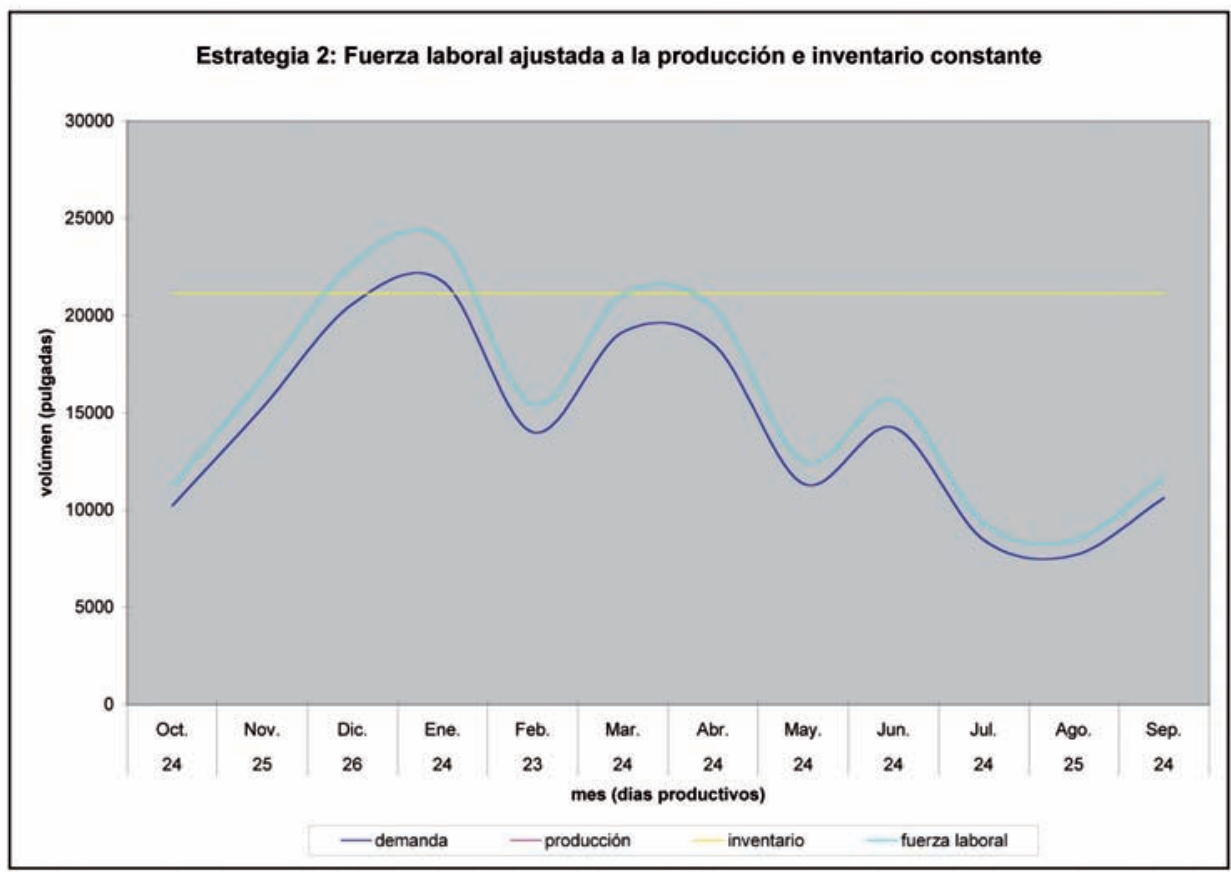

Figura 4. Gráfico Plan 2. 


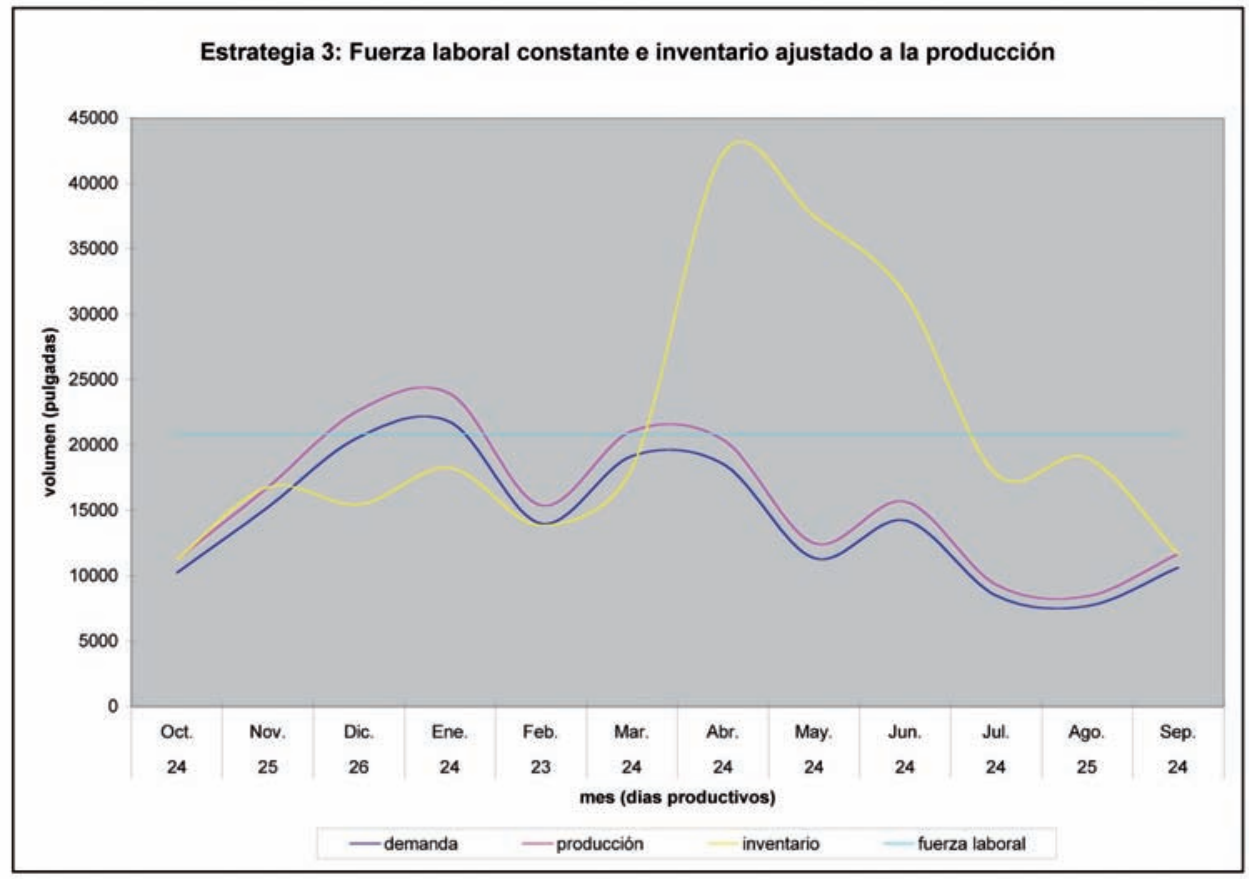

Figura 5. Gráfico Plan 3.

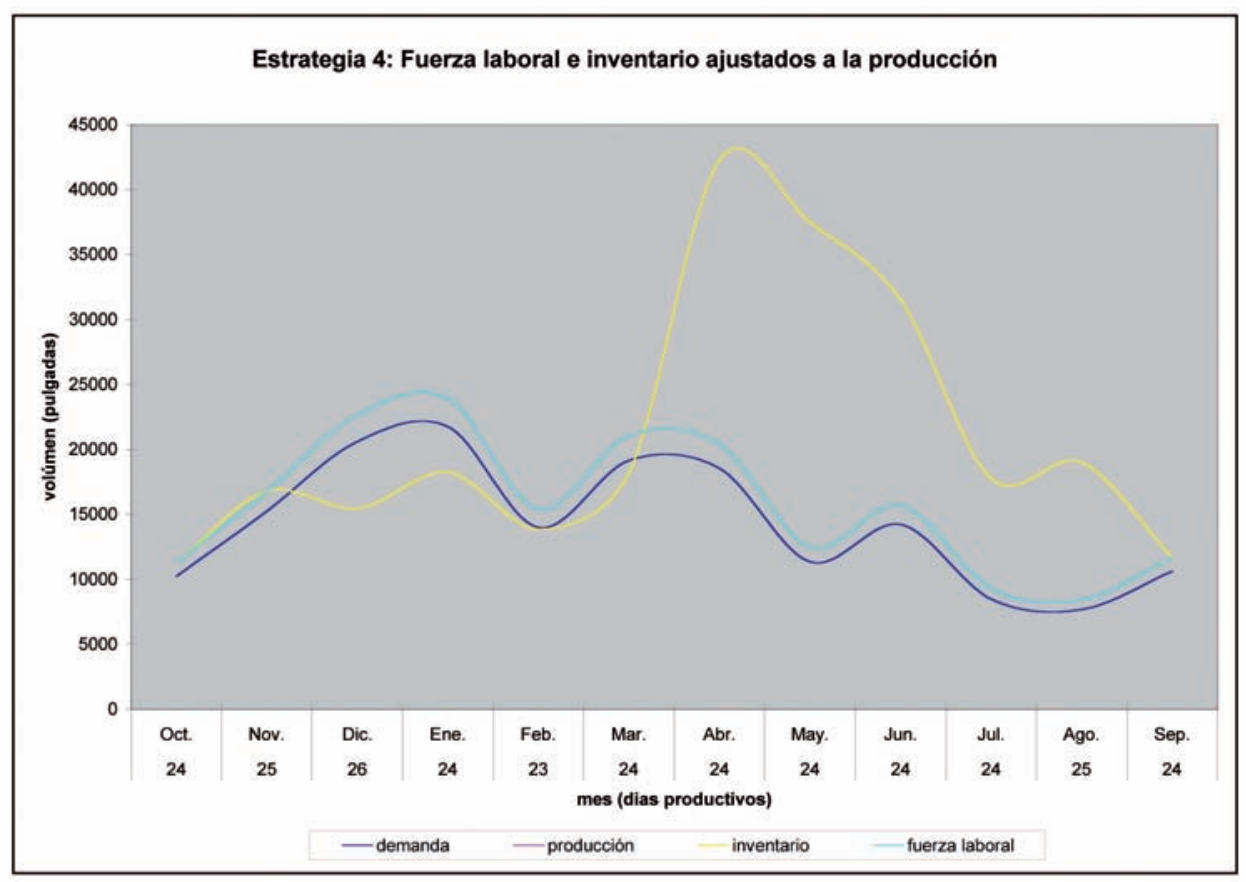

Figura 6. Gráfico plan 4.

Observación: La curva de producción es idéntica a la de fuerza laboral. 
Las tablas $10 ; 11 ; 12$ y 13 dan cuenta de los costos asociados a cada plan propuesto evaluado.

Tabla 10. Costos pertinentes Plan 1 (miles \$).

\begin{tabular}{|c|c|c|c|c|c|c|c|c|c|c|c|c|c|}
\hline \multirow{2}{*}{ Ítems } & \multicolumn{13}{|c|}{ MES } \\
\hline & Oct. & Nov. & Dic. & Ene. & Feb. & Mar. & Abr. & May. & Jun. & Jul. & Ago. & Sep. & Total \\
\hline Administración & 1.909 & 1.909 & 1.909 & 1.909 & 1.909 & 1.909 & 1.909 & 1.909 & 1.909 & 1.909 & 1.909 & 1.909 & 22.911 \\
\hline Mat. Prima & 14.073 & 20.992 & 28.339 & 29.919 & 19.253 & 26.337 & 25.482 & 15.617 & 19.583 & 11.652 & 10.563 & 14.605 & 236.413 \\
\hline E. Eléctrica & 533 & 796 & 1.074 & 1.134 & 730 & 998 & 966 & 952 & 742 & 442 & 400 & 554 & 8.960 \\
\hline Grúas & 435 & 649 & 876 & 925 & 595 & 815 & 788 & 483 & 606 & 360 & 327 & 452 & 7.312 \\
\hline Insumos & 88 & 131 & 176 & 186 & 120 & 164 & 159 & 97 & 122 & 73 & 66 & 91 & 1.471 \\
\hline Mantención & 272 & 405 & 547 & 578 & 372 & 508 & 492 & 301 & 378 & 225 & 204 & 282 & 4.564 \\
\hline Herramientas & 66 & 9 & 132 & 139 & 90 & 123 & 119 & 73 & 91 & 54 & 49 & 68 & 1.101 \\
\hline $\begin{array}{l}\text { Mano de Obra: } \\
\text { 1. Estable }\end{array}$ & 1.434 & 1.434 & 1.434 & 1.434 & 1.434 & 1.434 & 1.434 & 1.434 & 1.434 & 1.434 & 1.434 & 1.434 & 17.211 \\
\hline 2. Eventual & 0 & 0 & 0 & 0 & 0 & 0 & 0 & 0 & 0 & 0 & 0 & 0 & 0 \\
\hline 3. Horas extras & 0 & 0 & 340 & 433 & 0 & 188 & 97 & 0 & 0 & 0 & 0 & 0 & 1.058 \\
\hline 4. Bonos & 0 & 0 & 0 & 0 & 0 & 0 & 0 & 0 & 0 & 0 & 0 & 0 & 0 \\
\hline 5. Subcontratos & 472 & 704 & 951 & 1.004 & 646 & 883 & 855 & 524 & 657 & 354 & 490 & 490 & 7.930 \\
\hline 6. Contratos & 0 & 0 & 0 & 0 & 0 & 0 & 0 & 0 & 0 & 0 & 0 & 0 & 0 \\
\hline 7. Despidos & 0 & 0 & 0 & 0 & 0 & 0 & 0 & 0 & 0 & 0 & 0 & 0 & 0 \\
\hline Inventarios & 316 & 170 & 205 & 132 & 247 & 133 & 1.005 & 751 & 436 & 144 & 111 & 305 & 3.956 \\
\hline
\end{tabular}

Tabla 11. Costos pertinentes Plan 2 (miles $\$)^{3}$

\begin{tabular}{|c|c|c|c|c|c|c|c|c|c|c|c|c|c|}
\hline \multirow{2}{*}{ Ítems } & \multicolumn{13}{|c|}{ MES } \\
\hline & Oct. & Nov. & Dic. & Ene. & Feb. & Mar. & Abr. & May. & Jun. & Jul. & Ago. & Sep. & Total \\
\hline Administración & 1.909 & 1.909 & 1.909 & 1.909 & 1.909 & 1.909 & 1.909 & 1.909 & 1.909 & 1.909 & 1.909 & 1.909 & 22.911 \\
\hline Mat. Prima & 14.073 & 20.992 & 28.339 & 29.919 & 19.253 & 26.337 & 25.482 & 15.617 & 19.583 & 11.652 & 10.563 & 14.605 & 236.413 \\
\hline E. Eléctrica & 533 & 796 & 1.074 & 1.134 & 730 & 998 & 966 & 952 & 742 & 442 & 400 & 554 & 8.960 \\
\hline Grúas & 435 & 649 & 876 & 925 & 595 & 815 & 788 & 483 & 606 & 360 & 327 & 452 & 7.312 \\
\hline Insumos & 88 & 131 & 176 & 186 & 120 & 164 & 159 & 97 & 122 & 73 & 66 & 91 & 1.471 \\
\hline Mantención & 272 & 405 & 547 & 578 & 372 & 508 & 492 & 301 & 378 & 225 & 204 & 282 & 4.564 \\
\hline Herram & 66 & 2 & 132 & 139 & 90 & 123 & 119 & 7 & 8 & 54 & 49 & 68 & 1.101 \\
\hline $\begin{array}{l}\text { Mano } \\
\text { 1. Esta }\end{array}$ & 830 & 1.299 & 1.434 & & & & & & 1.199 & & & 854 & 3.307 \\
\hline 2. Eventual & 0 & 0 & $\begin{array}{c}1.434 \\
0\end{array}$ & $\begin{array}{c}1.434 \\
0\end{array}$ & 0 & $\begin{array}{c}1 .+34 \\
0\end{array}$ & $\begin{array}{c}1.404 \\
0\end{array}$ & $\begin{array}{c}800 \\
0\end{array}$ & 0 & 0 & $\begin{array}{c}0 \\
0\end{array}$ & $\begin{array}{c}004 \\
0\end{array}$ & 0 \\
\hline 3. Horas extras & 0 & 0 & 340 & 433 & 0 & 188 & 97 & 0 & 0 & 0 & 0 & 0 & 1.058 \\
\hline 4. Bonos & 0 & 0 & 0 & 0 & 0 & 0 & 0 & 0 & 0 & 0 & 0 & 0 & 0 \\
\hline 5. Subcontratos & 472 & 704 & 951 & 1.004 & 646 & 883 & 855 & 524 & 657 & 391 & 354 & 490 & 7.930 \\
\hline 6. Contratos & 0 & 450 & 150 & 0 & 0 & 150 & 0 & 0 & 450 & 0 & 0 & 150 & 1.350 \\
\hline 7. Despidos & 0 & 0 & 0 & 0 & 300 & 0 & 0 & 1.200 & 0 & 900 & 300 & 0 & 2.700 \\
\hline Inventarios & 316 & 170 & 205 & 132 & 247 & 133 & 1.005 & 751 & 436 & 144 & 111 & 305 & 3.956 \\
\hline
\end{tabular}

3 La tabla 11 se elaboró con las mismas consideraciones aplicadas para el plan 1. El costo unitario por pulgada producida $=$ costo total anual $/$ volumen anual producido $=1.655 \$ /$ pulg. 
Tabla 12. Costos pertinentes Plan $3(\text { miles } \$)^{4}$

\begin{tabular}{|c|c|c|c|c|c|c|c|c|c|c|c|c|c|}
\hline \multirow{2}{*}{ Ítems } & \multicolumn{13}{|c|}{ MES } \\
\hline & Oct. & Nov. & Dic. & Ene. & Feb. & Mar. & Abr. & May. & Jun. & Jul. & Ago. & Sep. & Total \\
\hline Administración & 1.909 & 1.909 & 1.909 & 1.909 & 1.909 & 1.909 & 1.909 & 1.909 & 1.909 & 1.909 & 1.909 & 1.909 & 22.911 \\
\hline Mat. Prima & 14.073 & 20.992 & 28.339 & 29.919 & 19.253 & 26.337 & 25.482 & 15.617 & 19.583 & 11.652 & 10.563 & 14.605 & 236.413 \\
\hline E. Eléctrica & 533 & 796 & 1.074 & 1.134 & 730 & 998 & 966 & 952 & 742 & 442 & 400 & 554 & 8.960 \\
\hline Grúas & 435 & 649 & 876 & 925 & 595 & 815 & 788 & 483 & 606 & 360 & 327 & 452 & 7.312 \\
\hline Insumos & 88 & 131 & 176 & 186 & 120 & 164 & 159 & 97 & 122 & 73 & 66 & 91 & 1.471 \\
\hline Mantención & 272 & 405 & 547 & 578 & 372 & 508 & 492 & 301 & 378 & 225 & 204 & 282 & 4.564 \\
\hline Herramientas & 66 & 98 & 132 & 139 & 90 & 123 & 119 & 73 & 91 & 54 & 49 & 68 & 1.101 \\
\hline $\begin{array}{l}\text { Mano de Obra: } \\
\text { 1. Estable }\end{array}$ & 1.434 & 1.434 & 1.434 & 1.434 & 1.434 & 1.434 & 1.434 & 1.434 & 1.434 & 1.434 & 1.434 & 1.434 & 17.211 \\
\hline 2. Eventual & 0 & 0 & 0 & 0 & 0 & 0 & 0 & 0 & 0 & 0 & 0 & 0 & 0 \\
\hline 3. Horas extras & 0 & 0 & 340 & 433 & 0 & 188 & 97 & 0 & 0 & 0 & 0 & 0 & 1.058 \\
\hline 4. Bonos & 0 & 0 & 0 & 0 & 0 & 0 & 0 & 0 & 0 & 0 & 0 & 0 & 0 \\
\hline 5. Subcontratos & 472 & 704 & 951 & 1.004 & 646 & 883 & 855 & 524 & 657 & 354 & 490 & 490 & 7.930 \\
\hline 6. Contratos & 0 & 0 & 0 & 0 & 0 & 0 & 0 & 0 & 0 & 0 & 0 & 0 & 0 \\
\hline 7. Despidos & 0 & 0 & 0 & 0 & 0 & 0 & 0 & 0 & 0 & 0 & 0 & 0 & 0 \\
\hline Inventarios & 30 & 44 & 41 & 48 & 37 & 48 & 112 & 99 & 83 & 47 & 50 & 31 & 669 \\
\hline
\end{tabular}

Tabla 13. Costos pertinentes Plan $4(\text { miles } \$)^{5}$

\begin{tabular}{|c|c|c|c|c|c|c|c|c|c|c|c|c|c|}
\hline \multirow{2}{*}{ Ítems } & \multicolumn{13}{|c|}{ MES } \\
\hline & Oct. & Nov. & Dic. & Ene. & Feb. & Mar. & Abr. & May. & Jun. & Jul. & Ago. & Sep. & Total \\
\hline Administración & 1.909 & 1.909 & 1.909 & 1.909 & 1.909 & 1.909 & 1.909 & 1.909 & 1.909 & 1.909 & 1.909 & 1.909 & 22.911 \\
\hline Mat. Prima & 14.073 & 20.992 & 28.339 & 29.919 & 19.253 & 26.337 & 25.482 & 15.617 & 19.583 & 11.652 & 10.563 & 14.605 & 236.413 \\
\hline E. Eléctrica & 533 & 796 & 1.074 & 1.134 & 730 & 998 & 966 & 952 & 742 & 442 & 400 & 554 & 8.960 \\
\hline Grúas & 435 & 649 & 876 & 925 & 595 & 815 & 788 & 483 & 606 & 360 & 327 & 452 & 7.312 \\
\hline Insumos & 88 & 131 & 176 & 186 & 120 & 164 & 159 & 97 & 122 & 73 & 66 & 91 & 1.471 \\
\hline Mantención & 272 & 405 & 547 & 578 & 372 & 508 & 492 & 301 & 378 & 225 & 204 & 282 & 4.564 \\
\hline Herram & 66 & 98 & 132 & 139 & 90 & 123 & 119 & 73 & 91 & 54 & 49 & 68 & 1.101 \\
\hline $\begin{array}{l}\text { Mano d } \\
\text { 1. Estah }\end{array}$ & 830 & 1.299 & 1.434 & 1.434 & 1.434 & 1.434 & 1.434 & 860 & 1.199 & 722 & 613 & 854 & 13.307 \\
\hline 2. Eventual & 0 & 0 & 0 & 0 & 0 & 0 & 0 & 0 & 0 & 0 & 0 & 0 & 0 \\
\hline 3. Horas extras & 0 & 0 & 340 & 433 & 0 & 188 & 97 & 0 & 0 & 0 & 0 & 0 & 1.058 \\
\hline 4. Bonos & 0 & 0 & 0 & 0 & 0 & 0 & 0 & 0 & 0 & 0 & 0 & 0 & 0 \\
\hline 5. Subcontratos & 472 & 704 & 951 & 1.004 & 646 & 883 & 855 & 524 & 657 & 391 & 354 & 490 & 7.930 \\
\hline 6. Contratos & 0 & 450 & 150 & 0 & 0 & 150 & 0 & 0 & 450 & 0 & 0 & 150 & 1.350 \\
\hline 7. Despidos & 0 & 0 & 0 & 0 & 300 & 0 & 0 & 1.200 & 0 & 900 & 300 & 0 & 2.700 \\
\hline Inventarios & 30 & 44 & 41 & 48 & 37 & 48 & 112 & 99 & 83 & 47 & 50 & 31 & 669 \\
\hline
\end{tabular}

4 La tabla se elaboró con las mismas consideraciones aplicadas para los planes 1 y 2 . Costo unitario por pulgada producida $=$ costo total anual $/$ volumen anual producido $=1.637 \$ /$ pulg .

5 La tabla se elaboró con las mismas consideraciones aplicadas para los planes 1,2 y 3 . Costo unitario por pulgada producida $=$ costo total anual $/$ volumen anual producido $=1.638 \$ /$ pulg. 


\section{DISCUSIÓN}

Se evalúan los cuatro planes propuestos sobre la base del costo total durante el horizonte a planificar, lo que se presenta en la tabla 14.

Tabla 14. Comparación de los planes en base a sus costos

\begin{tabular}{|c|c|c|c|c|}
\hline \multirow{2}{*}{ Ítems } & \multicolumn{4}{|c|}{ Costos anuales para cada plan } \\
\hline & Plan $1(\$)$ & Plan $2(\$)$ & Plan $3(\$)$ & Plan $4(\$)$ \\
\hline Administración & 22.910 .604 & 22.910 .604 & 22.910 .604 & 22.910 .604 \\
\hline Mat. Prima & 236.413 .375 & 236.413 .375 & 236.413 .375 & 236.413 .375 \\
\hline E. Eléctrica & 8.960 .214 & 8.960 .214 & 8.960 .214 & 8.960 .214 \\
\hline Grúas & 7.311 .982 & 7.311 .982 & 7.311 .982 & 7.311 .982 \\
\hline Insumos & 1.471 .059 & 1.471 .059 & 1.471 .059 & 1.471 .059 \\
\hline Mantención & 4.563 .535 & 4.563 .535 & 4.563 .535 & 4.563 .535 \\
\hline Herramientas & 1.101 .497 & 1.101 .497 & 1.101 .497 & 1.101 .497 \\
\hline $\begin{array}{l}\text { Mano de Obra: } \\
\text { 1. Estable }\end{array}$ & 17.210 .880 & 13.307 .390 & 17.210 .880 & 13.307 .390 \\
\hline 2. Eventual & 0 & 0 & 0 & 0 \\
\hline 3. Horas extras & 1.058 .250 & 1.058 .250 & 1.050 .250 & 1.058 .250 \\
\hline 4. Bonos & 0 & 0 & 0 & 0 \\
\hline 5. Sub contratos & 7.930 .103 & 7.930 .103 & 7930.103 & 7.930 .103 \\
\hline 6. Contratos & 0 & 1.350 .000 & 0 & 1.350 .000 \\
\hline 7. Despidos & 0 & 2.700 .000 & 0 & 2.700 .000 \\
\hline Inventarios & 3.955 .785 & 3.955 .785 & 668.523 & 668.523 \\
\hline TOTAL & 312.887 .283 & 313.033 .793 & 309.600 .021 & 309.746 .531 \\
\hline
\end{tabular}

Los costos proyectados no varían por la tasa de producción, ya que se utiliza la misma para las cuatro estrategias, las cuales se obtienen de la demanda del período referencial, más un inventario de seguridad del $10 \%$.

En los ítems administración, materia prima, energía eléctrica, grúas, insumos, mantención y herramientas, no se produce ninguna modificación en los costos, por lo que en este caso reciben el nombre de costos inmóviles.

En el ítem mano de obra, el costo total para satisfacer las necesidades de producción es prácticamente despreciable entre estrategias, ya que por la combinación entre los niveles de producción y el número de empleados necesarios para estos niveles, el costo de trabajar con personal estable se equipara a la suma de los costos de empleados variables, más los costos de contratación y despidos; sí es importante considerar la distribución de los costos dentro de este ítem. Para efectos prácticos, cabe mencionar que éstos se ven influenciados por el criterio del planificador respecto a la ponderación que le otorga a ciertos ítems.

Por su parte, el ítem inventario afecta al costo total de producción, principalmente producto de la estacionalidad climática de la región, donde existen meses en el año en que sólo se pueden manejar inventarios de un menor volumen que en otros períodos, donde se debe compatibilizar la producción con la necesidad de mantener la madera en el proceso de secado por más tiempo. 
Comparados los costos de los planes propuestos, se optó por el que presenta los costos operativos menores a lo largo de un horizonte de doce meses, teniendo además en consideración que la comparación entre estrategias se hace en función del costo como criterio fundamental en la toma de decisiones, pero además asumiendo los compromisos adquiridos por la empresa a fin de mantener a su personal en forma estable. Por ello, la planeación agregada propuesta a elegir corresponde al Plan 3 "Fuerza laboral constante e inventario ajustado a la producción".

Finalmente, este estudio de caso permite aconsejar el uso de la PA en pequeños aserraderos, ya que es de fácil aplicación, toda vez que la reducción del problema, el uso de una base de datos simple, el apoyo computacional y las capacidades profesionales destinadas a la planificación, concomitan positivamente en la gestión de la empresa.

\section{CONCLUSIONES}

La PA aplicada en la empresa permitió reducir en un 1,92\% los costos de producción, manteniendo una fuerza laboral permanente como parte de su política interna de responsabilidad empresarial.

El plan agregado propuesto para la barraca de maderas en estudio contempló una fuerza laboral constante y un inventario ajustado a la producción. Se estableció la tasa de producción agregada mensual para el periodo proyectado en 189.130,7 pulgadas anuales. La producción agregada anual se distribuye entre los meses de Octubre a Septiembre del año siguiente en los siguientes volúmenes: 11.259; 16.794; 22.671; $23.935 ; 15.402 ; 21.069 ; 20.385 ; 12.494 ; 15.666 ; 9.321 ; 8.450$ y 11.684 pulgadas.

El nivel de la fuerza laboral queda propuesto según el plan aprobado, en 9 personas como personal estable en producción directa, lo que corresponde a 20.736 horas hombre anuales, más 850 horas extras y 6.304 horas hombre por concepto de subcontratos.

El nivel de inventarios propuesto corresponde a 21.131 pulgadas mensuales promedio. La distribución mensual de los inventarios para el período proyectado corresponde a: $11.259 ; 16.794 ; 15.457 ; 18.236$; $13.862 ; 18.196 ; 42.298 ; 37.481 ; 31.517 ; 17.772 ; 19.022$ y 11.684 pulgadas.

El costo por unidad producida con el plan propuesto es de 1.637 \$ pulg. Respecto del nivel de la fuerza laboral, la diferencia monetaria que se presentó entre una fuerza laboral estable y una fuerza laboral ajustada a la producción fue de $\$ 12.209$ mensuales, por lo que se puede concluir que la variable fuerza laboral para una empresa de estas características es considerable desde una perspectiva social más que económica.

El nivel de inventarios, en cambio, se consideró significativo para una empresa pequeña como ésta, ya que la diferencia de costos entre un inventario constante y un inventario ajustado a la producción es de \$273.931 mensuales.

La comparación económica entre el período referencial y cada uno de los 4 planes alternativos analizados entregó, en cada caso, costos unitarios de producción inferiores en el plan respecto al período referencial, lo que ratifica la importancia de la planeación. 


\section{BIBLIOGRAFÍA}

Adam, E.; Ebert, R. 1991. Administración de la producción y las operaciones. México. Prentice-Hall Hispanoamérica S.A. $4^{\circ}$ edición. 739 p.

Bredstrom, D.; Lundgren, J.; Ronnquist, M.; Carlsonn, D.; Mason, A. 2004. Supply chain optimization the pull mill industry - IP models, column generation and novel constraint branches. European Journal of Operation Research 156 (1): 2-22

Buxey, G. 2005. Aggregate planning for seasonal demand; reconciling theory with practice. International Journal of Operations \& Production Management 25 (11): 1083 - 1100.

Buxley, G. 2003. Strategic not tactics drive aggregate planning. International Journal of Production Economics 85 (3): 331 - 346.

Chase, R.; Alquilano, N.; Jacobs, F. 2002. Administración de producción y operaciones. $8^{\mathrm{a}}$ edición. Santa Fe de Bogotá. Mc Graw Hill. 869 p.

Gunasekaran, A.; Marri, H. 2004. Application of aggregate production planning in developing countries. International Journal of Computer Applications in Technology 20 (4): 172-179.

Heizer, J.; Render, B. 2001. Dirección de la producción. Madrid. Prentice Hall. $6^{\circ}$ edición. 451 p.

Horngren, C.; Sundem, G. 1994. Contabilidad administrativa. México. Prentice Hall Hispanoamericana S.A. $9^{\circ}$ edición. 920 p.

Jingsheng, J. 1999. Mathematical models for maximizing aggregate plan production. Journal of Construction Engineering and Management 125 (1): 53 - 60.

Martel, A.; Rizk, N.; D'amours, S.; Bouchriha, H. 2004. Synchronized production-distribution planning in the pulp and paper industry. Working paper. Research Consortium on e - Business in the Forest Products Industry. Network Organization Technology Research Center. Université Laval, Québec. Canadá.

Monks, J. 1992. Administración de operaciones. México. Mc Graw Hill. 411 p.

Mula, J.; Poler, R.; García, J. 2006. Evaluación de sistemas para la planificación y control de la producción. Información Tecnológica 17(1): 19-34.

Noori, H.; Radford, R. 1997. Administración de operaciones y producción. Santa Fe de Bogotá. Mc Graw Hill. 648 p.

Pradenas, L.; Peñailillo, F.; Ferland, J. 2004. Aggregate production planning problem. A new algorithmic. Electronic Note in Discrete Mathematics 18: 193 - 199.

Sapag, N.; Sapag, R. 1996. Preparación y evaluación de proyectos. $3^{\text {a }}$ edición. Santa Fe de Bogotá. Mc Graw Hill. 404 p.

Schroeder, R. 1992. Administración de operaciones. México. Mc Graw Hill. Interamericana de México. $3^{\mathrm{a}}$ edición. $885 \mathrm{p}$.

Welsch, G.; Hilton, R.; Gordon, P.; Rivera, C. 2005. Presupuestos, planificación y control. México. Prentice Hall, 6 a edición. 474 p. 